\title{
Novos limitantes lagrangeanos para o Problema Probabilístico de Localização-Alocação de Máxima Cobertura utilizando grafos de cobertura
}

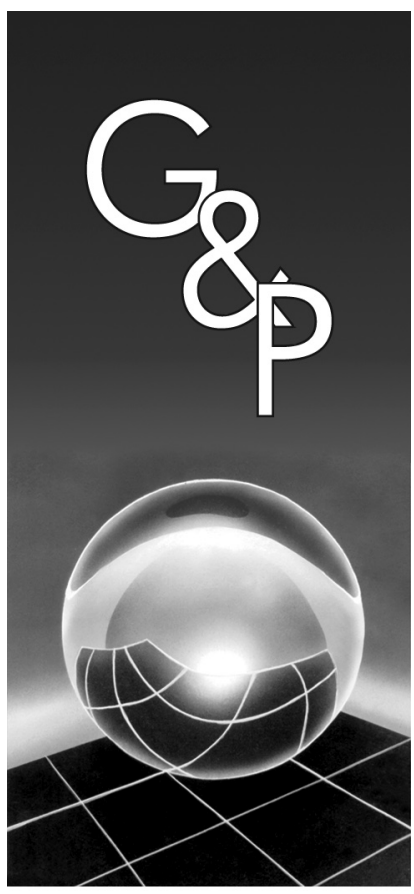

Francisco de Assis Corrêa

Luiz Antonio Nogueira Lorena

Glaydston Mattos Ribeiro

Resumo

O Problema Probabilístico de Localização-Alocação de Máxima Cobertura (PPLAMC) consiste em localizar facilidades, maximizando a população atendida e fornecendo um bom nível de serviço para toda a população, ou seja, deve-se garantir que um usuário, ao chegar a um centro, não espere mais que um tempo máximo permitido ou não encontre uma fila de atendimento com um número de usuário maior que um valor máximo. Estes dois parâmetros dependem da taxa de chegada dos usuários e do atendimento, ambos probabilísticos. Devido às dificuldades intrínsecas do problema, neste artigo são discutidos limitantes lagrangeanos para o PPLAMC obtidos com a relaxação lagrangeana com clusters (LagClus). Na sua proposição inicial, a LagClus utilizou um grafo de conflitos, porém neste artigo esta relaxação foi aplicada em um grafo especial denominado grafo de cobertura.

Palavras-chave: Relaxação lagrangeana. Relaxação lagrangeana com clusters. Problemas de localização. Máxima cobertura.

\section{Introdução}

Os problemas de localização têm como objetivo localizar facilidades para atender aos usuários que estão espacialmente distribuídos. As facilidades são centros que fornecem algum tipo de serviço. Por exemplo, podem-se citar hospitais que fornecem serviços médicos a seus pacientes e bancos que gerenciam as contas de seus clientes.

Muitos desses problemas são combinatoriais e, mesmo com aplicativos sofisticados como o CPLEX (ILOG, http:// www.ilog.com) e o XPRESS (DASH OPTIMIZATION, http://www.dashoptimization.com), soluções ótimas podem ser difíceis de serem encontradas. Portanto, muitos pesquisadores recorrem a heurísticas, metaheurísticas, métodos de decomposição e relaxações, para obter soluções primais de boa qualidade ou até mesmo limitantes duais interessantes que permitam avaliar soluções factíveis.

O Problema de Localização de Máxima Cobertura PLMC tem sido consideravelmente tratado na literatura desde a sua formulação feita por Church e ReVelle (1974). Esse problema busca obter a configuração para localizar uma quantidade pré-definida de facilidades que atenda o maior número de indivíduos de uma população, considerada uma dada distância ou um tempo padrão do ponto de demanda. Não se busca com este modelo atender toda a população, mas oferecer o máximo de atendimento, considerando os recursos disponíveis. A Figura 1 mostra três facilidades localizadas que atendem a vários pontos de demanda, porém, note que alguns pontos não estão cobertos pelos centros. Normalmente na literatura, um ponto é considerado coberto se ele está dentro de uma dada distância ou de um dado tempo da facilidade.

Vários modelos aplicados a uma grande faixa de problemas, nos setores público e privado, são extensões dessa formulação, adaptados para melhor representar a realidade. As aplicações variam de sistemas de emergência (EATON et al., 1986; CURRENT; O'KELLY, 1992), serviços hierárquicos de saúde (MOORE; REVELLE, 1982), controle de poluição do ar (HOUGLAND; STEPHENS, 1976), e sistemas congestionados (MARIANOV; SERRA, 1998; 2001). Os métodos de solução para o PLMC incluem relaxação de programação linear (CHURCH; REVELLE, 1974), heurísticas gulosas (DASKIN, 1995), relaxação lagrangeana (GALVÃO; REVELLE, 1996), heurística lagrangeana/ surrogate (PEREIRA; LORENA, 2002), geração de colunas (PEREIRA et al., 2007), dentre outros. Considerável revisão deste tema pode ser encontrada em Chung (1986), Hale, Moberg (2003), Serra, Marianov (2004), Galvão (2004). 
Em muitas aplicações práticas do PLMC, a distância ou o tempo entre os pontos de demanda e as facilidades constituem fatores importantes para estabelecer o nível de serviço oferecido aos usuários. Por outro lado, sabe-se que as chegadas dos usuários em sistemas de atendimento, como hospitais e bancos, são regidas por processos aleatórios e que, muitas vezes, proporcionam um congestionamento e a geração de filas (FOGLIATTI; MATTOS, 2007). Sistemas que apresentam tal comportamento indicam que o nível de serviço prestado aos usuários não pode ser medido apenas levando-se em consideração a distância ou o tempo entre os pontos de demanda e as facilidades, mas também os atributos de fila como o comprimento e o tempo de espera. Sendo assim, os centros devem ser localizados de tal maneira que os usuários cheguem dentro de um tempo aceitável e também que, uma vez na fila, o comprimento desta não seja maior que um valor máximo e/ou o tempo de espera não seja maior que um limite máximo (MARIANOV; SERRA, 1998).

Os congestionamentos acontecem quando o centro não é capaz de atender a sua demanda. Modelos determinísticos do PLMC consideram capacidades limites para os centros, entretanto, a própria dinâmica de chegada dos usuários muitas vezes impossibilita o uso desses modelos. Além disso, os próprios atendentes nos centros possuem um processo de atendimento que não é constante, com isso, modelos determinísticos podem apresentar servidores com excesso de trabalho ou até mesmo ociosos, pois se

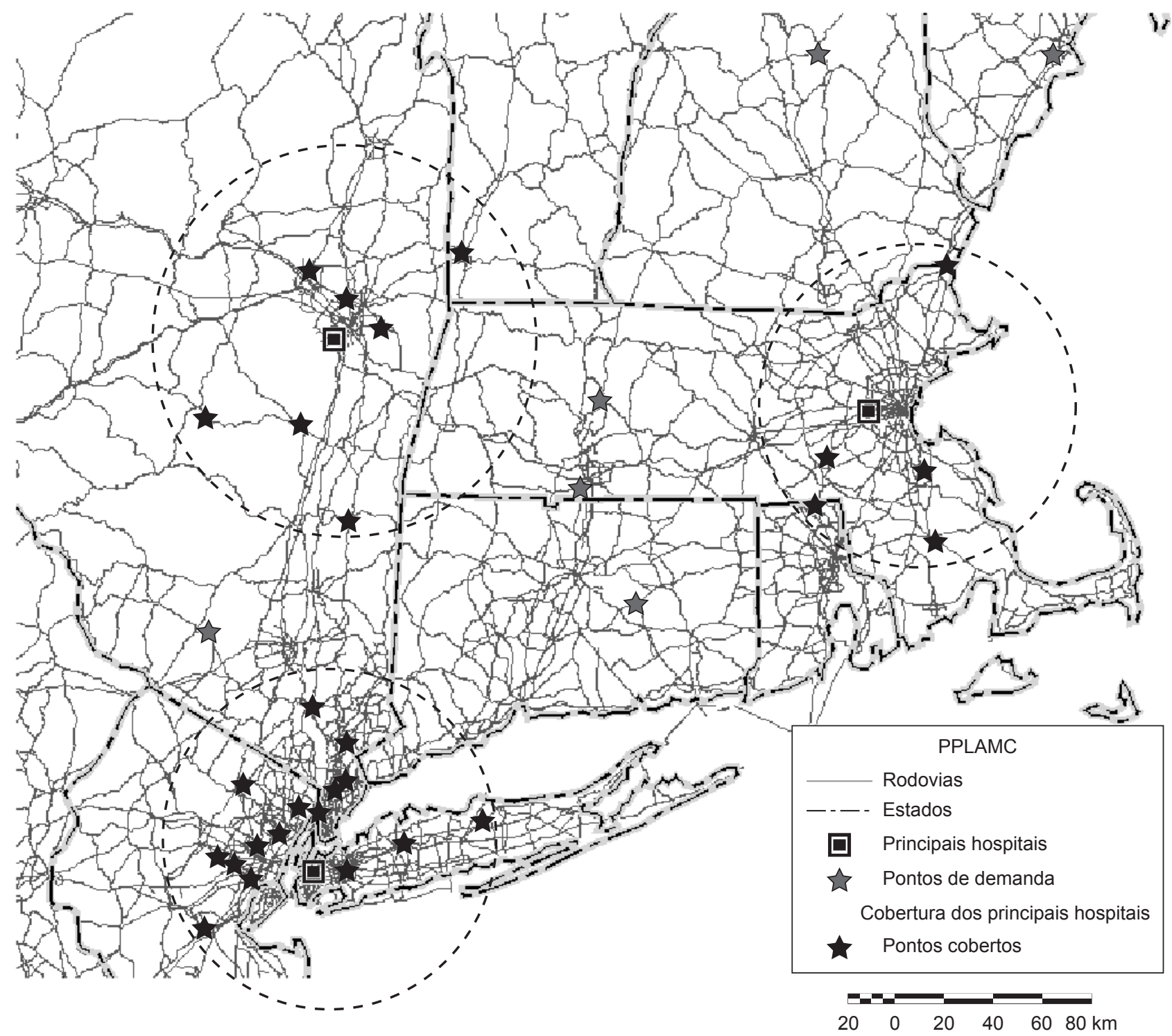

Figura 1. O Problema da Localização de Máxima Cobertura - PLMC. 
considera um tempo de atendimento médio (MARIANOV; SERRA, 1998; 2001).

Considerando então essa aleatoriedade no processo de chegada e atendimento, Marianov e Serra (1998) propuseram modelos matemáticos em que a estocasticidade da demanda é explicitamente considerada nas restrições de capacidade, que, ao invés de serem limitadas a um máximo, têm um limite mínimo definido pelos autores para a qualidade dos seus serviços. Essa qualidade é refletida no tempo de espera e/ou na quantidade de pessoas que aguardam $o$ atendimento.

Marianov e Serra (1998) definiram então o Problema Probabilístico de Localização-Alocação de Máxima Cobertura (PPLAMC) que busca localizar uma dada quantidade de facilidades com um ou vários servidores, de modo que a população, a uma distância padrão do centro, seja servida adequadamente, isto é, que ninguém fique na fila por um período maior que um dado tempo limite, e que um usuário, ao chegar ao centro, não encontre um número de outros clientes acima do previsto, com probabilidade maior ou igual a dado valor definido $a$ priori. Os autores trabalharam com postos de saúde,

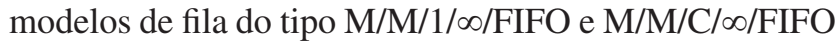
(LARSON; ODONI, 1981) e taxas de chegadas a cada centro $j$ distribuídas conforme uma Poisson com taxa $\lambda_{j} \mathrm{e}$ tempo de atendimento exponencialmente distribuído com taxa média $\mu_{j}$ para cada servidor.

Considerando a dificuldade embutida desses problemas, este artigo apresenta limitantes lagrangeanos para o PPLAMC. São apresentados limitantes lagrangeanos com a relaxação proposta por Corrêa, Lorena (2006), e segundo a Relaxação Lagrangeana com Clusters (LagClus) proposta por Ribeiro, Lorena (2007). A LagClus, inicialmente proposta, utiliza uma representação do problema por meio de um grafo de conflitos que é particionado em clusters. Em seguida, as arestas de conexão são relaxadas no sentido lagrangeano e os subproblemas podem ser então independentemente resolvidos. Neste artigo apresenta-se a LagClus sobre um grafo especial denominado grafo de cobertura. $\mathrm{O}$ particionamento deste grafo produz um número menor de restrições a serem relaxadas. Resultados computacionais mostram que a LagClus com o grafo cobertura apresenta bons limitantes.

O restante do artigo está distribuído como segue: na Seção 2 é apresentada a formulação tradicional do PPLAMC e a formulação proposta por Cornuéjols e Thizy (1982) da qual se visualiza o grafo de conflitos; na Seção 3 são apresentados os principais passos da LagClus e como os grafos de conflitos e de cobertura, bem como os seus particionamentos, podem ser obtidos; na Seção 4 são apresentados os resultados computacionais obtidos neste artigo; e por último, a Seção 5 apresenta as principais conclusões e recomendações do trabalho.

\section{Formulações para o problema probabilístico de localização-alocação de máxima cobertura}

O modelo tradicional PLMC proposto por Church e ReVelle (1974) não pode ser usado para tratar as restrições de congestionamento, pois não contém variáveis de alocação, o que impede de computar as solicitações de serviços que chegam a um centro, e, consequentemente, de determinar quando ocorre um congestionamento.

A modelagem matemática proposta para PPLAMC foi escrita como um problema tipo $p$-medianas (LORENA, 2003), modificado para comportar as variáveis de localização e alocação, tendo como objetivo maximizar a população coberta, considerando uma determinada quantidade de centros de atendimento (facilidades).

Seja $I$ o conjunto dos pontos de demanda a serem alocados e $N_{i}$ o conjunto de localizações candidatas que estão dentro de uma dada distância do ponto $i$. Formalmente, as alocações são representadas pelas variáveis binárias $x_{i j}, \forall i \in I$ e $j \in N_{i}$. Com isso, $x_{i j}=1$, se o ponto de demanda $i$ for alocado ao centro $j, x_{i j}=0$, caso contrário. As localizações são representadas pelas variáveis binárias $y_{j}$, com $y_{j}=1$, se o centro $j$ for selecionado e $y_{j}=0$, caso contrário. Todo ponto de demanda é um potencial centro de atendimento.

Assim, a formulação do PPLAMC pode ser escrita como (MARIANOV; SERRA, 1998):

$$
v(P P L A M C)=\operatorname{MAX}\left\{\sum_{i \in I} \sum_{j \in N_{i}} a_{i} x_{i j}\right\}
$$

(PPLAMC) Sujeito a:

$$
\begin{gathered}
x_{i j} \leq y_{j} \quad \forall i \in I \text { e } j \in N_{i} \\
\sum_{j \in N_{i}} x_{i j} \leq 1 \quad \forall i \in I \\
\sum_{i \in I} f_{i} x_{i j} \leq \mu_{j} \sqrt[b+2]{1-\alpha} f \quad \forall j \\
\sum_{i \in I} f_{i} x_{i j} \leq \mu_{j}+\frac{1}{\tau} \ln \left(1-\alpha_{f}\right) \quad \forall j \\
\sum_{i \in I} y_{i}=p \\
y_{j} \text { e } x_{i j} \in\{0,1\} \quad \forall i \in I \text { e } j \in N_{i}
\end{gathered}
$$


em que:

- $a_{i}$ representa a população total do ponto de demanda $i$

- $b$ é o número máximo de usuários na fila com probabilidade de, no mínimo, $\alpha_{f}$;

- $\tau$ é o tempo máximo de espera na fila com probabilidade de, no mínimo, $\alpha_{f}$;

- $f_{i}$ é a taxa de chegadas dos usuários conforme um processo de Poisson;

- $\mu_{j}$ é a taxa média de atendimento em que o tempo de atendimento está exponencialmente distribuído; e

- $p$ é o número de facilidades a serem localizadas.

A função objetivo descrita em (1) indica que a população total coberta pelos $p$ centros deve ser a maior possível. As restrições definidas em (2) garantem que só é possível alocar um ponto de demanda $i$ a um centro $j$ se houver um centro em $j$. As restrições descritas em (3) garantem que cada ponto de demanda deve ser alocado a, no máximo, um centro.

As restrições descritas em (4a) e (4b) estão associadas à questão do comprimento máximo da fila e ao tempo máximo de atendimento, respectivamente. Utiliza-se uma delas, dependento da restrição que se quer tratar. As definidas por (4a) garantem que, com probabilidade $\alpha_{f}$, cada centro tenha no máximo $b$ pessoas na fila, por outro lado, as definidas em (4b) garantem que, com probabilidade $\alpha_{f}$ o tempo de atendimento em cada centro seja de no máximo $\tau$. A restrição definida em (5) garante que $p$ centros serão selecionados, e as restrições descritas em (6) garantem que todas as variáveis são binárias.

As restrições descritas em (4a) e (4b) foram definidas por Marianov e Serra (1998) para o modelo de fila M/M/1/œ/ FIFO, ou seja, os intervalos entre chegadas são exponenciais, o tempo de atendimento é exponencial, com 1 servidor, sem limite de capacidade e disciplina de fila do tipo "o primeiro a chegar é o primeiro a ser atendido" (first in - first out).

Usuários chegam a um centro $j$ provenientes de um ponto de demanda $i$ com uma taxa de chegada $f_{i}$. Como em um centro $j$, os usuários podem vir de vários pontos de demanda $i$, faz-se necessário levantar a taxa de chegada geral ao centro $j$. Dessa maneira, considerando que as chegadas dos usuários de todos os pontos de demanda $i$ acontecem de maneira superposta, sem perda de generalidade, a taxa de chegada ao centro $j$ pode ser assim definida (MARIANOV; SERRA, 1998; CORRÊA; LORENA; 2006): $\lambda_{j}=\sum_{i \in I} f_{i} x_{i j}$.

Para que se tenha equilíbrio no processo de atendimento dos centros, faz-se necessário que $\mu_{j} \geq \lambda_{j}$ (FOGLIATTI; MATTOS, 2007).
Como indicam Marianov e Serra (1998), Corrêa e Lorena (2006), o lado direito das equações (4a) e (4b) são constantes, calculados para $\mu_{j}, \alpha_{\rho} b$ e $\tau$, definidos a priori. Simplificando, essas restrições podem ser substituídas, respectivamente, pelos dois conjuntos de restrições a seguir, em que $K_{\mu b \alpha_{f}}^{j}=\mu_{j} \sqrt[b+2]{1-\alpha_{f}}$ e $K_{\mu \tau \alpha_{f}}^{j}=\mu_{j}+\frac{1}{\tau} \ln \left(1-\alpha_{f}\right)$

$$
\begin{array}{ll}
\sum_{i \in I} f_{i} x_{i j} \leq K_{\mu b \alpha_{f}}^{j} & \forall j \\
\sum_{i \in I} f_{i} x_{i j} \leq K_{\mu \tau \alpha_{f}}^{j} & \forall j
\end{array}
$$

Considerando as instâncias de grande porte (324 e 818 pontos de demanda) propostas por Corrêa e Lorena (2006) e Corrêa et al. (2006a) disponíveis em http://www.lac.inpe. br/ lorena/instancias.html, o CPLEX 10 (ILOG, 2006), com a formulação mostrada acima, não consegue resolver todas as instâncias, parando várias vezes por falta de memória. Consequentemente buscaram-se relaxações lagrangeanas que pudessem fornecer limitantes duais.

O modelo proposto por Marianov e Serra (1998) descrito anteriormente pode ser alterado para comportar restrições de adjacência ou de conflitos. Uma restrição de conflito garante que no máximo uma variável receberá o valor 1 (ATAMTÜRK et al., 2000). Considerando então o complemento das variáveis de localização, ou seja, $\bar{y}_{j}=1-y_{j}$ conforme indicado por Cornuéjols e Thizy (1982), o seguinte modelo pode ser escrito:

$$
v(\overline{P P L A M C})=\operatorname{MAX}\left\{\sum_{i \in I} \sum_{j \in N_{i}} a_{i} x_{i j}\right.
$$

$(\overline{P P L A M C})$ Sujeito a (3), (6) e (7a) ou (7b),

$$
\begin{gathered}
x_{i j}+\bar{y}_{j} \leq 1 \quad \forall i \in I \text { e } j \in N_{i} \\
\sum_{i \in I} \bar{y}_{i}=|N|-p
\end{gathered}
$$

em que $N=\cup_{i \in I} N_{i}$.

Com esse modelo, é possível construir o grafo de conflitos para a LagClus.

\section{Relaxação lagrangeana com clusters a partir de grafos de conflitos e de cobertura}

O conjunto de restrições de adjacências descrito em (3) e em (9) permite explorar LagClus que vem se mostrando forte para alguns problemas de otimização: Carregamento de Paletes (RIBEIRO; LORENA, 2007), Rotulação Cartográfica (RIBEIRO; LORENA, 2008b), Estivagem de Unidades de Celulose (RIBEIRO; LORENA, 2008a), 
Problema de Localização de Facilidades Não Capacitado (CORRÊA et al, 2006b).

A idéia da LagClus consiste em dividir o problema original em pequenos subproblemas (clusters) que podem ser independentemente resolvidos, e relaxar, no sentido lagrangeano, as restrições que se apresentam nas interligações dos clusters.

Ribeiro, Lorena (2007; 2008a; 2008b) vêm aplicando a LagClus a partir da representação do problema por meio de grafos de conflitos. Segundo Atamtürk et al. (2000), um grafo de conflitos representa uma relação lógica entre variáveis binárias. Existe um vértice para cada variável binária e uma aresta (restrição de conflito) entre dois vértices quando no máximo uma das variáveis representadas pode receber o valor um. Agora note que as restrições apresentadas em (3) e (9) são restrições de conflito, Consequentemente um grafo de conflitos pode ser obtido para o PPLAMC, e a LagClus pode ser então aplicada.

Ribeiro, Lorena (2007) definem alguns passos para se usar a LagClus:

- Montar o grafo de conflitos $G=(V, A)$ do problema;

- Aplicar uma heurística de particionamento para dividir o grafo $G$ em $Q$ clusters com aproximadamente a mesma cardinalidade, obtendo-se um $Q$-particionamento. O problema passa a ser então representado por meio de sua função objetivo, sujeita às restrições de adjacências (arestas) divididas em dois conjuntos: um com restrições de adjacência intra-clusters; e outro com restrições de adjacência entre-clusters ou arestas de ligação;
- Relaxar, no sentido lagrangeano, as restrições de adjacências entre-clusters; e

- Decompor a relaxação lagrangeana resultante em $Q$ subproblemas e resolvê-la por meio de um algoritmo de subgradientes.

A seguir, é apresentado como o grafo de conflitos pode ser obtido para o PPLAMC bem como o grafo de cobertura.

\subsection{Grafo de conflitos para o problema probabilístico de localização-alocação de máxima cobertura}

Considere os dados da Tabela 1 que indicam quatro pontos de demanda com suas respectivas coordenadas de localização. Admitindo que cada ponto de demanda possa ser um centro e que um ponto de demanda $j$ está coberto por um centro $i$ se este estiver a uma distância euclidiana menor ou igual a 4, a coluna Pontos Cobertos apresenta os pontos de demanda cobertos por cada um dos possíveis centros. Levando-se em consideração o segundo modelo mostrado para o PPLAMC, as restrições definidas em (3) e (9) devem ser escritas e um grafo de conflitos pode ser gerado, veja Figura 2.

Considerando agora o segundo passo da LagClus, suponha que o grafo de conflitos tenha sido particionado em 2 clusters conforme mostra a Figura 2. Nessa figura, as linhas mais espessas mostradas à direita indicam quais restrições devem ser relaxadas (neste exemplo, restrições definidas conforme (9)).

Ao considerar o PPLAMC e o particionamento mostrado na Figura 2, além das restrições de conflitos já identificadas previamente para serem relaxadas, algumas restrições do tipo (7a) ou (7b) e a restrição (10) também devem ser

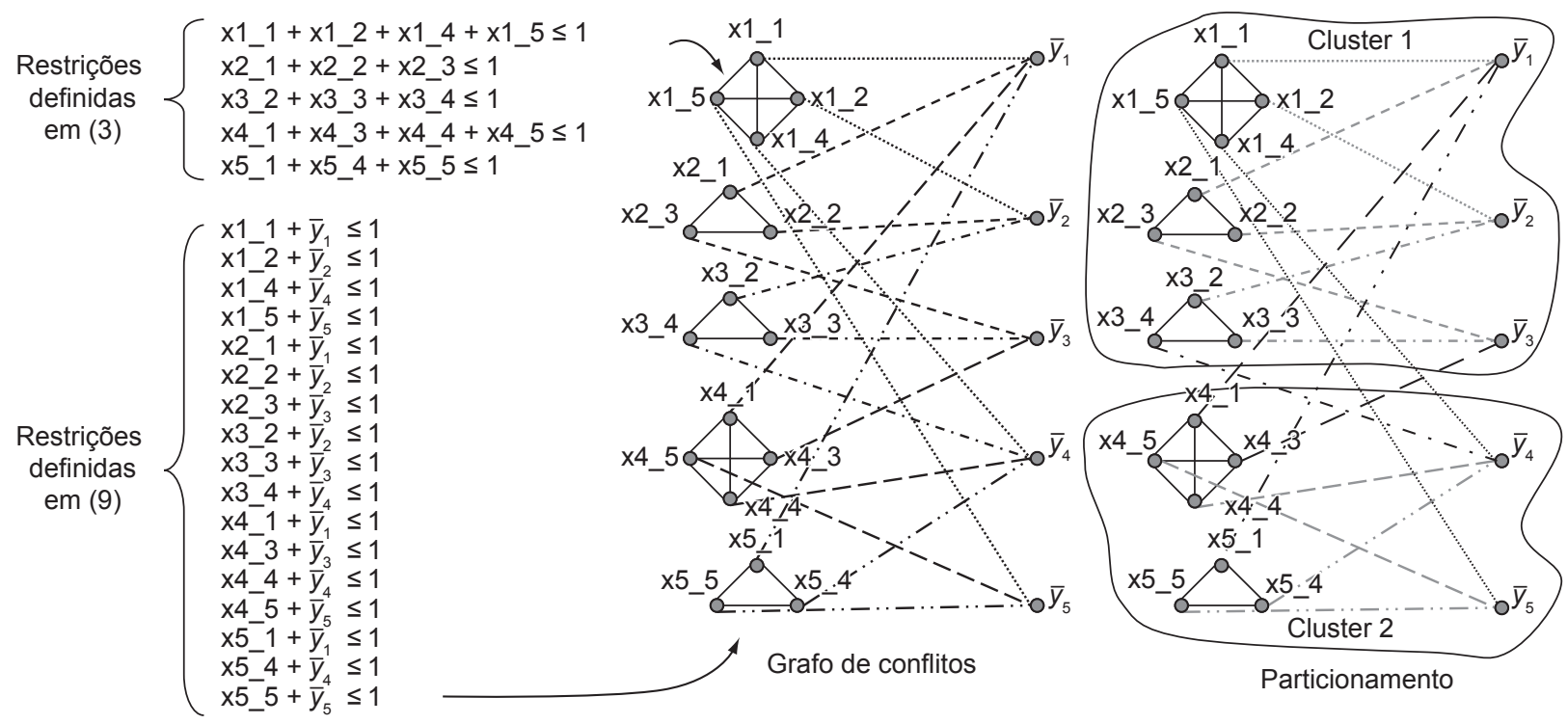

Figura 2. Exemplo de um grafo de conflitos. 
relaxadas do PPLMAC. Ao escrever o modelo matemático todo para o exemplo, percebe-se que 11 restrições são relaxadas: 6 estão mostradas Figura 2, 4 são restrições do tipo (7a) ou 7(b) e a restrição (10).

Resultados computacionais mostraram que a decomposição do grafo de conflitos do PPLAMC em clusters, relaxa muitas restrições o que gera limitantes duais de baixa qualidade. Isso motivou o estudo do particionamento dos grafos de cobertura ao invés dos grafos de conflitos. $\mathrm{O}$ grafo de cobertura é obtido a partir da primeira formulação do PPLAMC proposta por Marianov, Serra (1998).

\subsection{Grafo de cobertura para o problema probabilístico de localização-alocação de máxima cobertura}

Um grafo de cobertura $G=(V, A)$ apresenta um conjunto $V$ de vértices formados pelos locais candidatos e um conjunto de $A$ de arestas que indica a cobertura de cada ponto de demanda. Esse grafo também apresenta clusters de vértices, logo a LagClus pode ser aplicada.

Para demonstrar como obtê-lo, considere novamente os dados da Tabela 1. A partir dos dados apresentados, sabe-se que os conjuntos de localizações candidatas de cada ponto são: $N_{1}=\{1,2,4,5\}, N_{2}=\{1,2,3\}, N_{3}=\{2,3,4\}$, $N_{4}=\{1,3,4,5\}$ e $N_{5}=\{1,4,5\}$. Sendo assim, a Figura 3 apresenta a cobertura de todos os pontos e por fim, o grafo de cobertura do problema.

Particionando o grafo de cobertura em dois clusters como realizado na Figura 2, ou seja, $y_{1}, y_{2}$ e $y_{3}$ no Cluster 1 e $y_{4}$ e $y_{5}$ no Cluster 2 (Veja Figura 3), e considerando a formulação de Marianov e Serra (1998), as seguintes restrições devem ser relaxadas: $x_{1 \_1}+x_{1 \_2}+x_{11_{4}}+x_{1 \_5} \leq 1$, $x_{3 \_2}+x_{3 \_3}+x_{3 \_4} \leq 1, x_{4 \_1}+x_{4-3}+x_{4 \_4}+x_{4-5} \leq 1$ e $x_{5-1}+$ $x_{54}+x_{55} \leq 1$, além da restrição (5). Isso resulta em apenas cinco restrições relaxadas.

Para comparar o número de restrições relaxadas por cada um dos métodos, grafos de conflitos e de cobertura foram obtidos para três conjuntos de instâncias propostos na literatura. $\mathrm{O}$ primeiro $\left(\mathrm{C}_{1}\right)$ foi proposto por Marianov e Serra (1998) e contém problemas com 30 pontos, o segundo e o terceiro $\left(\mathrm{C}_{2}\right.$ e $\left.\mathrm{C}_{3}\right)$ foram propostos por Corrêa e Lorena (2006) e Corrêa et al. (2007) e possuem, respectivamente, 324 e 818 pontos. O particionamento do grafo foi realizado pela heurística METIS (KARYPIS;

Tabela 1. Exemplo de um problema de máxima cobertura com raio de cobertura igual a 4 .

\begin{tabular}{cccc}
\hline Pontos de Demanda & X & Y & Pontos Cobertos \\
\hline 1 & 4 & 8 & $1,2,4,5$ \\
2 & 7 & 10 & $1,2,3$ \\
3 & 8 & 7 & $2,3,4$ \\
4 & 5 & 5 & $1,3,4,5$ \\
5 & 2 & 6 & $1,4,5$ \\
\hline
\end{tabular}

KUMAR, 1998) que é uma heurística bem conhecida para particionamento de grafos. O METIS busca particionar o grafo reduzindo o número de restrições com vértices em diferentes clusters e mantendo aproximadamente clusters de mesmo tamanho. Os experimentos foram realizados variando-se o número de clusters para uma melhor comparação. Os resultados estão mostrados na Tabela 2.

Analisando a Tabela 2, note que o particionamento do grafo de conflitos particiona um número menor de arestas que o particionamento do grafo de cobertura. Por outro lado, o número de restrições relaxadas no modelo com restrições de conflitos $(\overline{\text { PPLAMC }})$ é maior que o do outro modelo. Ao considerar os valores médios, o modelo $\overline{P P L A M C}$ relaxa aproximadamente 30 vezes mais restrições que o modelo PPLAMC. Esse comportamento justifica o uso da LagClus com o grafo de cobertura e a formulação original de Marianov e Serra (1998).

Considerando o particionamento do grafo de cobertura $G=(V, E)$ em $Q$ partes com vértices $V_{1}, V_{2}, \ldots, V_{Q}$, subgrafos $G_{q}=G\left[V_{q}\right]$ podem ser definidos bem como suas arestas $E_{q}=E\left(G_{q}\right)$. Com isso, as arestas que conectam os subgrafos, ou seja, as arestas particionadas, podem ser definidas como $\hat{E}=E \backslash \bigcup_{q=1}^{Q} E_{q}$. Desse modo, após a fase de particionamento, a formulação do PPLAMC pode ser novamente escrita (Equação 11):

$$
v(P P L A M C)=\operatorname{MAX} \sum_{q=1}^{Q} \mathbf{a}^{q} \mathbf{x}^{q}
$$

(PPLAMC) Sujeito a (Equações 12, 13 e 13a):

$$
\left[\begin{array}{llll}
A_{1} & A_{2} & \ldots & A_{Q}
\end{array}\right]\left(\begin{array}{c}
{\left[\begin{array}{c}
\boldsymbol{x} \\
\boldsymbol{y}
\end{array}\right]^{1}} \\
{\left[\begin{array}{c}
\boldsymbol{x} \\
\boldsymbol{y}
\end{array}\right]^{2}} \\
\vdots \\
{\left[\begin{array}{c}
\boldsymbol{x} \\
\boldsymbol{y}
\end{array}\right]^{Q}}
\end{array}\right) \sim \boldsymbol{b}
$$

$$
\left[\begin{array}{cccc}
B_{1} & 0 & 0 & 0 \\
0 & B_{2} & 0 & 0 \\
0 & 0 & \ddots & 0 \\
0 & 0 & 0 & B_{Q}
\end{array}\right]\left(\begin{array}{l}
{\left[\begin{array}{c}
\boldsymbol{x} \\
\boldsymbol{y}
\end{array}\right]^{1}} \\
{\left[\begin{array}{c}
\boldsymbol{x} \\
\boldsymbol{y}
\end{array}\right]^{2}} \\
\vdots \\
{\left[\begin{array}{c}
\boldsymbol{x} \\
\boldsymbol{y}
\end{array}\right]^{Q}}
\end{array}\right) \sim \boldsymbol{b}
$$




$$
\mathbf{x}^{q}, \mathbf{y}^{q} \in B^{\left|V_{q}\right|} \forall q \in\{1, . ., Q\}
$$

em que:

- $A_{\mathrm{q}}$ é uma matriz de coeficientes das restrições definidas em (3) associadas com as arestas $\hat{E}$, e da restrição definida em (5);

- $B_{q}$ é uma matriz de coeficientes das restrições definidas em (2), (4a), (4b) e das restrições definidas em (3) associadas às arestas de $E_{q}$;

- $\sim$ representa o operador relacional $=$ ou $\leq$ dependendo da respectiva restrição; e

- b é um vetor com os termos do lado direito das respectivas restrições.

Agora, relaxando no sentido lagrangeano as restrições de acoplamento dos blocos, ou seja, as restrições definidas em (12), cada subproblema $q$ pode ser assim escrito (Equação 14):

$v(P P L A M C)_{q}^{L G}=\operatorname{Max}\left\{\left(a^{q}-A_{q}^{T} \alpha\right)\left[\begin{array}{l}x \\ y\end{array}\right]^{q}: x^{q}, y^{q} \in C_{q}\right\}$

em que $\alpha$ é um vetor de multiplicadores lagrangeanos relacionados às restrições definidas em (12), e $C_{q}$ representa o conjunto de restrições embutido no cluster $q$, ou seja, o bloco $q$ das restrições definidas em (13).

Se $\alpha$ é um vetor de dimensão $K$ em que $\alpha_{k} \in \mathfrak{R}^{+}$ $\forall k=1, \ldots, K-1$ e $\alpha_{k} \in \Re$ tal que $K$ é o número de restrições de acoplamentos definidas em (12), o limitante lagrangeano pode ser assim escrito (Equação 15):
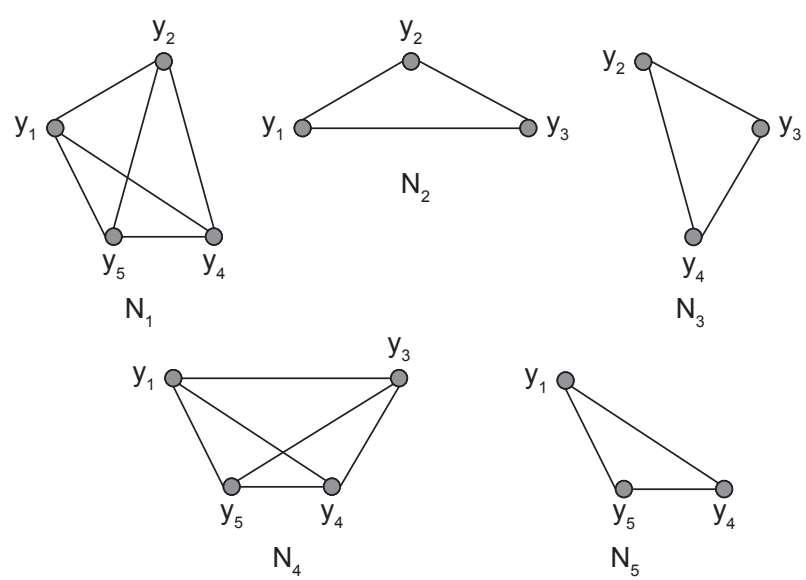

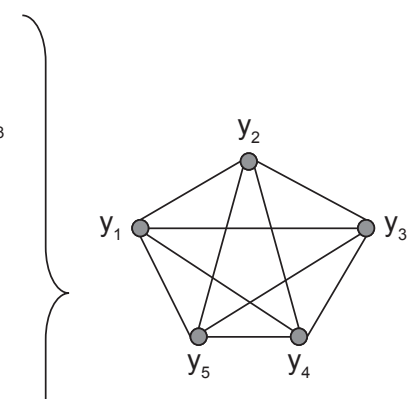

Grafo de cobertura

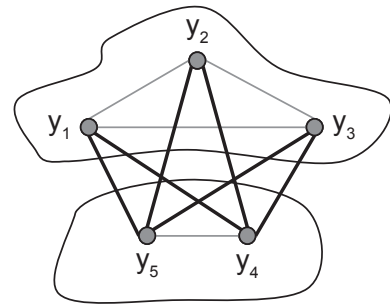

Particionamento

Figura 3. Exemplo de um grafo de cobertura.

Tabela 2. Número de restrições relaxadas na LagClus usando grafos de conflitos e de cobertura.

\begin{tabular}{|c|c|c|c|c|c|c|}
\hline \multirow[t]{2}{*}{ Conjunto* } & \multirow[t]{2}{*}{$\begin{array}{l}\mathrm{N}^{0} \text { de pontos } \\
\text { demanda }\end{array}$} & \multirow[t]{2}{*}{$\mathbf{N}^{0}$ de Clusters } & \multirow[t]{2}{*}{$\begin{array}{l}\text { Total de arestas } \\
\text { particionadas no } \\
\text { grafo de conflitos }\end{array}$} & \multirow[t]{2}{*}{$\begin{array}{l}\text { Total de arestas } \\
\text { particionadas no } \\
\text { grafo de cobertura }\end{array}$} & \multirow{2}{*}{$\begin{array}{c}\begin{array}{c}\text { Total de restrições } \\
\text { relaxadas pelo } \\
\text { modelo }\end{array} \\
\frac{P P L A M C}{}\end{array}$} & \multirow[t]{2}{*}{$\begin{array}{c}\text { Total de restrições } \\
\text { relaxadas pelo } \\
\text { modelo PPLAMC }\end{array}$} \\
\hline & & & & & & \\
\hline \multirow{3}{*}{$\mathrm{C}_{1}$} & 30 & 2 & 108 & 155 & 133 & 24 \\
\hline & & 4 & 224 & 261 & 253 & 30 \\
\hline & & 8 & 332 & 307 & 362 & 30 \\
\hline \multirow[t]{4}{*}{$\mathrm{C}_{2}$} & 324 & 5 & 361 & 1034 & 483 & 114 \\
\hline & & 10 & 742 & 2238 & 925 & 238 \\
\hline & & 20 & 1469 & 4186 & 1752 & 311 \\
\hline & & 30 & 2123 & 4364 & 2434 & 314 \\
\hline \multirow[t]{5}{*}{$\mathrm{C}_{3}$} & 818 & 5 & 11411 & 34457 & 11914 & 487 \\
\hline & & 10 & 21468 & 57579 & 22186 & 716 \\
\hline & & 20 & 36083 & 71157 & 36879 & 819 \\
\hline & & 30 & 42812 & 76283 & 43629 & 818 \\
\hline & & Média & 10648,45 & 22911,00 & 10995,45 & 354,64 \\
\hline
\end{tabular}

*Instâncias disponível em http://www.lac.inpe.br/ lorena/instancias.html. 
$v\left(P P L A M C^{L G}\right)=\sum_{q=1}^{Q}\left\{v(P P L A M C)_{q}^{L G}\right\}+\sum_{k=1}^{K-1} \alpha_{k}+p \alpha_{K}(15)$

\subsection{Experimentos computacionais}

Para os experimentos computacionais, como citado anteriormente, foram utilizadas as instâncias propostas por Marianov, Serra (1998), Corrêa, Lorena (2006a), Corrêa et al. (2007). Para uma melhor descrição bem como suas principais características, veja Corrêa e Lorena (2006a) e Corrêa et al. (2007). Para comparar os limitantes lagrangeanos obtidos com a LagClus, o CPLEX 10 (ILOG, 2006) foi utilizado. Todos os experimentos foram limitados em 3 horas de processamento.

Um algoritmo de subgradientes adaptado para a LagClus foi implementado conforme Parker e Rardin (1988), Narciso, Lorena (1999), Wolsey (1998). Os subproblemas da LagClus foram resolvidos usando o CPLEX 10 e, para o cálculo do passo, foram utilizadas as melhores soluções encontradas na literatura disponíveis nos trabalhos de Corrêa e Lorena (2006a) e Corrêa et al. (2007). O uso dessas melhores soluções se justifica uma vez que o objetivo deste trabalho é investigar os limitantes duais. Além disso, a relaxação Lagrangeana proposta por Corrêa e Lorena (2006a) foi implementada para comparação dos limitantes. Essa relaxação é aplicada nas restrições definidas em (3). O limitante de programação linear também foi calculado usando o CPLEX 10. Por último, todos os experimentos foram realizados em computador equipado com um Pentium IV 3.0 GHz com 1.0 GB de memória RAM.

As Tabelas 3, 4 e 5 apresentam os resultados encontrados neste artigo. A primeira coluna dessas tabelas apresenta o nome da instância que indica os valores de cinco parâmetros, sendo eles: tamanho da instância, $p, b, \mu$, $\varphi$ e $\tau$ : Por exemplo, a instância 324_20_0_2_95 indica que são 324 pontos, 20 centros (facilidades), que a fila está restringida por tamanho (se o parâmetro fosse 1 a fila estaria restringida pelo tempo), que existe um máximo de duas pessoas na fila com probabilidade de pelo menos $95 \%$. A coluna gap nessas tabelas é calculada da seguinte maneira:

$G A P(\%)=\frac{(\text { limitante superior }- \text { limitante inferior) }}{\text { limitante inferior }} \times 100$.

Em todas estas tabelas, nota-se que os limitantes de programação linear são altos se comparados com os do CPLEX, da Relaxação Lagrangeana de Corrêa e Lorena (2006a) e os da LagClus. As linhas em negrito indicam soluções ótimas encontradas pelos métodos.

Considere a Tabela 3. Para as instâncias apresentadas nessa tabela, o CPLEX apresenta bons resultados, resolvendo otimamente várias instâncias. A relaxação de programação linear apresenta um tempo computacional baixo, porém o gap é maior. Analisando as relaxações, percebe-se que a Relaxação Lagrangeana de Corrêa e Lorena (2006a) encontra várias soluções ótimas, enquanto a LagClus encontra apenas uma. Entretanto, ao se comparar os gaps médios, percebe-se que a LagClus apresenta um gap menor.

Considerando a Tabela 4, percebe-se que a Relaxação Lagrangeana de Corrêa e Lorena (2006a) não encontra nenhuma solução ótima e apresenta um gap médio maior que o do CPLEX e o da LagClus. Comparando agora o CPLEX e a LagClus, percebe-se que ambos resolveram de forma ótima 14 instâncias e que apresentam gaps e tempos computacionais médios da mesma ordem de grandeza.

Por último, a Tabela 5 apresenta os resultados para as instâncias de grande porte com 818 pontos. Observe que o gap médio da relaxação de programação linear é significativo, maior que $800 \%$. Por outro lado, a relaxação lagrangeana de Corrêa e Lorena (2006a) para essas instâncias não conseguiu executar a primeira iteração do algoritmo de subgradientes após 3 horas de processamento, Consequentemente nenhum gap pôde ser calculado. Analisando agora os resultados da LagClus, percebe-se que o tempo computacional médio utilizado por ela é comparável ao do CPLEX, além disso, a LagClus apresenta um gap médio menor que o do CPLEX e a LagClus resolveu otimamente a instância 818_20_1_41_85 não resolvida pelo CPLEX.

\section{Conclusões}

Este trabalho apresentou novos limitantes lagrangeanos para o Problema Probabilístico de Localização-Alocação de Máxima Cobertura (PPLAMC) obtidos com uma relaxação lagrangeana com clusters (LagClus) aplicada após a representação do PPLAMC por meio de um grafo de cobertura. A LagClus tem se mostrado mais forte que as relaxações tradicionais e esse comportamento novamente foi encontrado para o PPLAMC.

Para instâncias de grande porte, com 818 pontos, a LagClus apresentou gaps menores que o CPLEX $10 \mathrm{em}$ um tempo computacional médio semelhante. Além disso, a relaxação lagrangeana de Corrêa e Lorena (2006a) não apresentou limitantes para esses problemas.

Conforme indicam Ribeiro e Lorena (2007; 2008b), a decomposição do problema por meio de clusters permite a utilização da decomposição de Dantzig-Wolfe e, consequentemente, pode-se aplicar um algoritmo de geração de colunas (AGC). Os autores ainda indicam que o AGC apresenta resultados melhores que a LagClus, adicionando poucas colunas no problema mestre restrito. Atualmente encontra-se em desenvolvimento um AGC para o PPLAMC e resultados preliminares mostraram que os resultados da LagClus podem ser melhorados. Acredita-se que em breve novos limitantes duais e soluções primais sejam encontradas para o PPLAMC. 


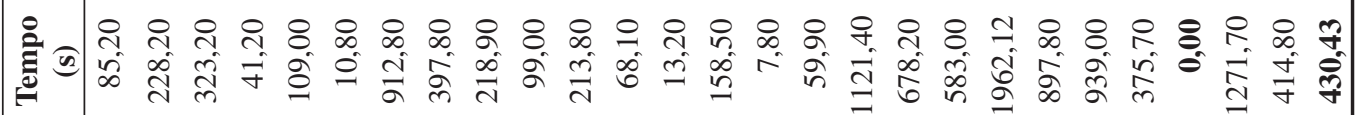
业

苞苛击

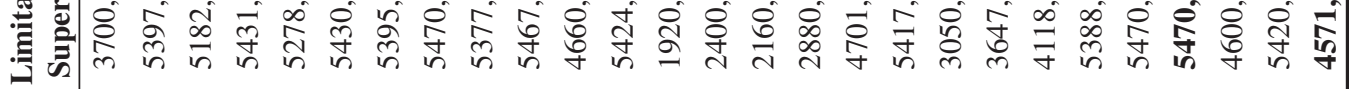

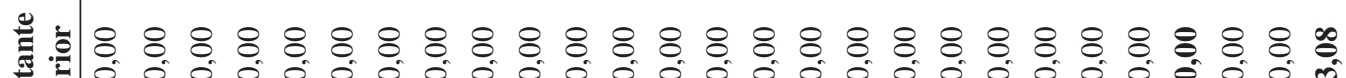

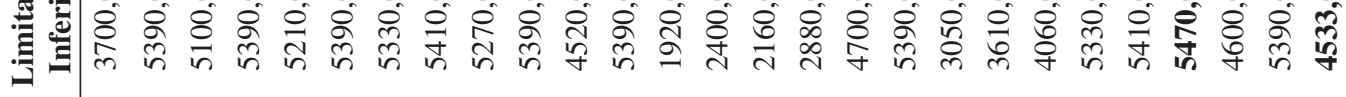

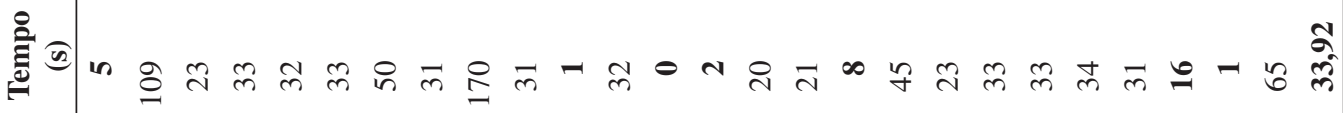

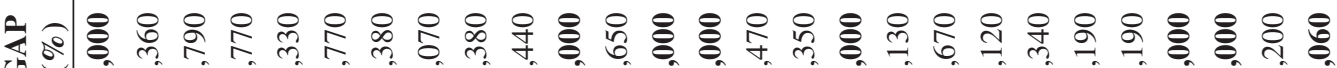

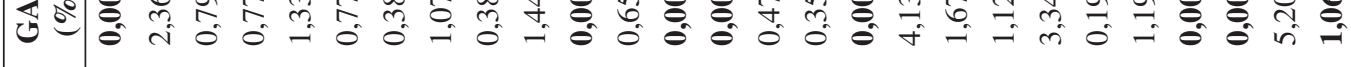

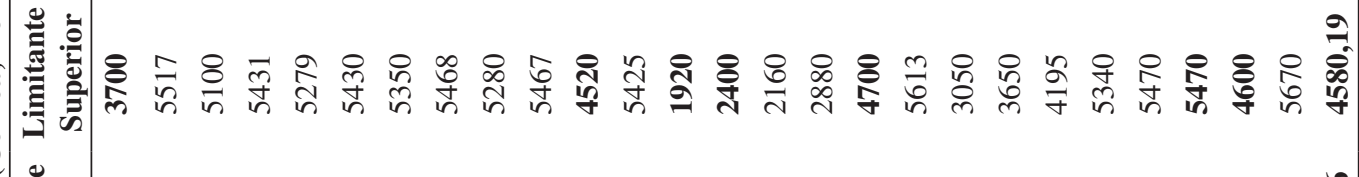

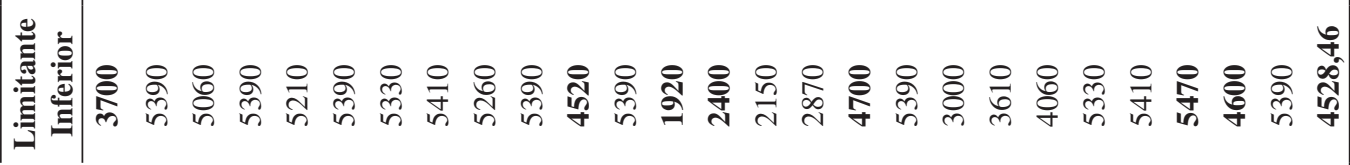

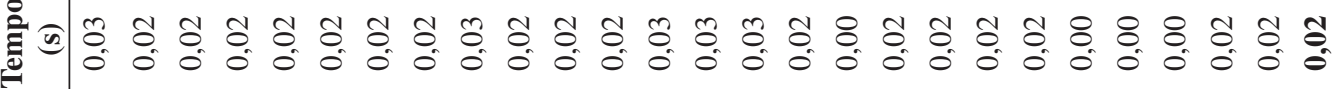

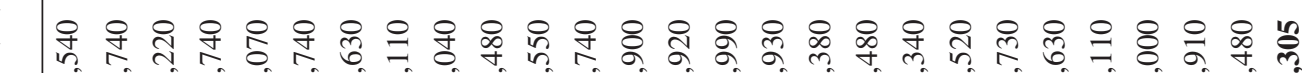

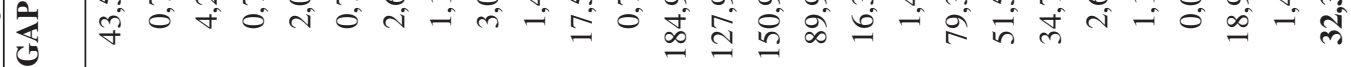

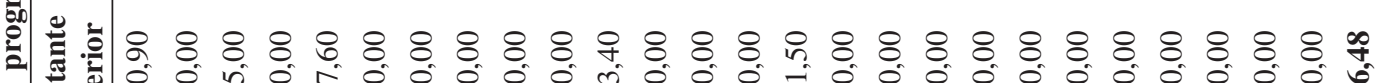

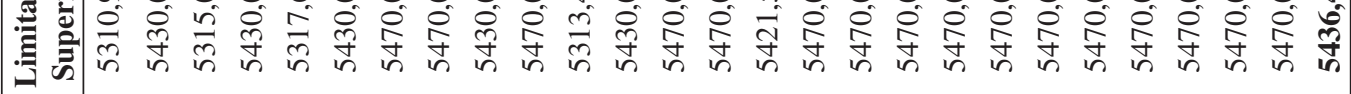

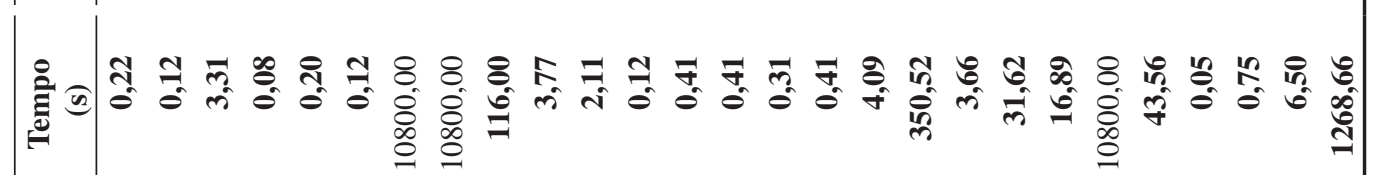
e

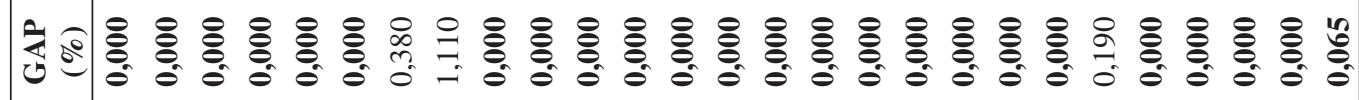

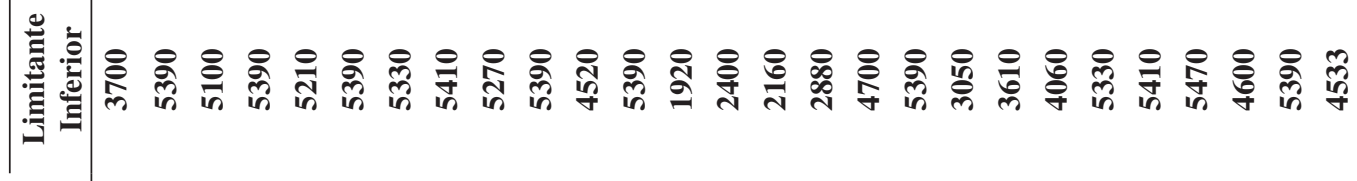

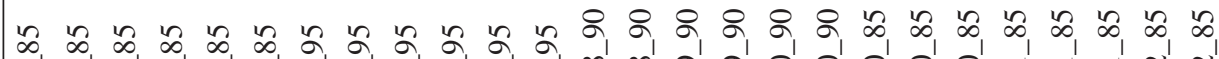

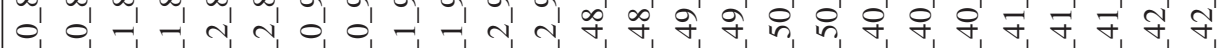

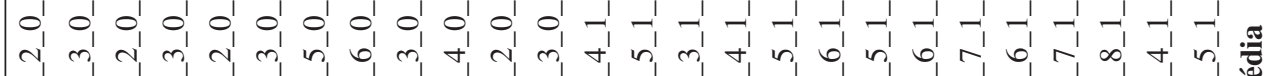

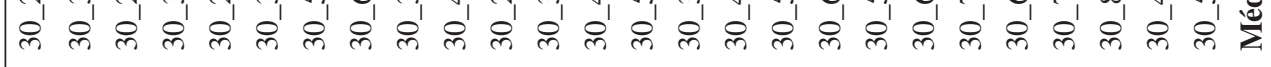




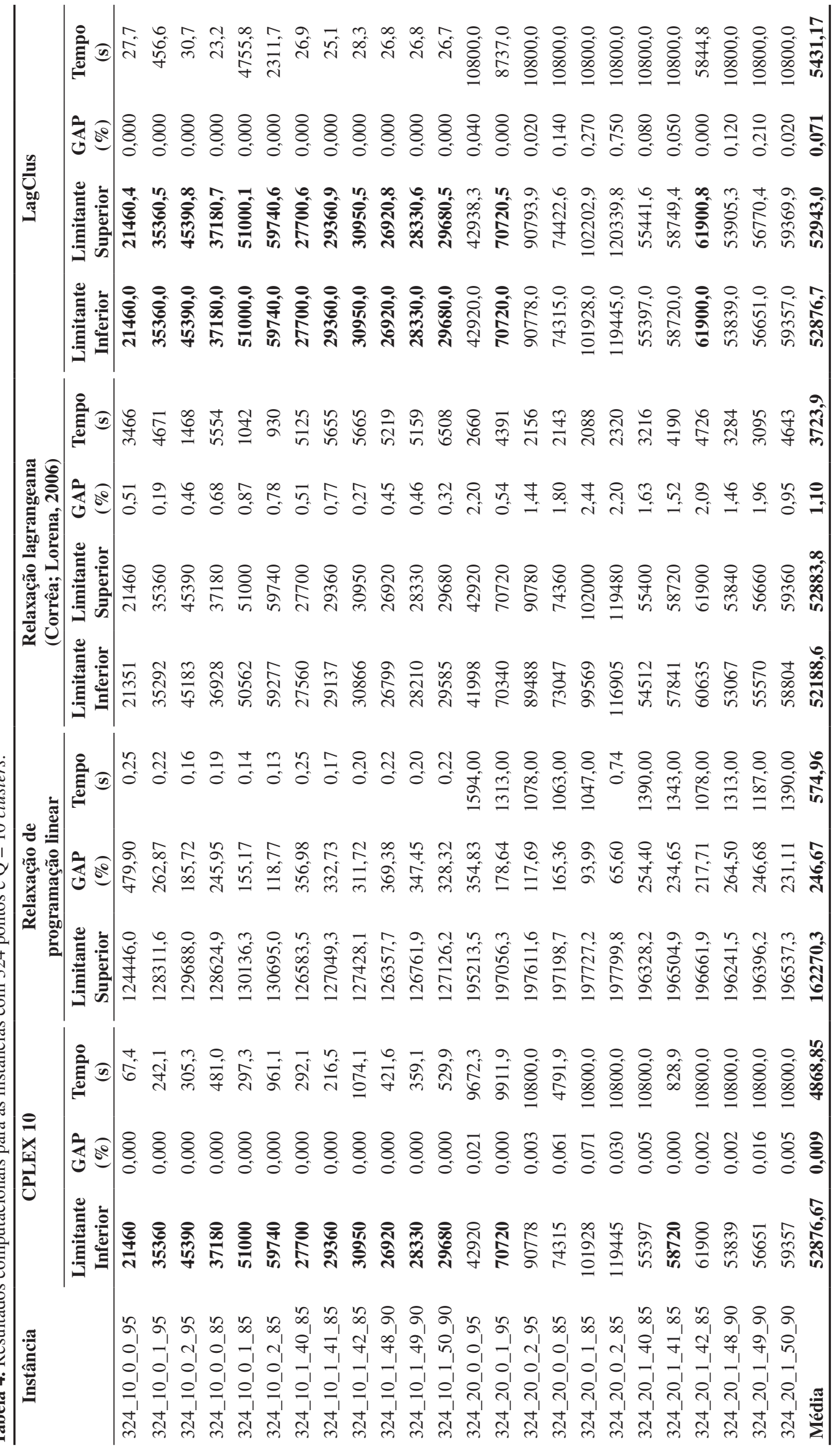




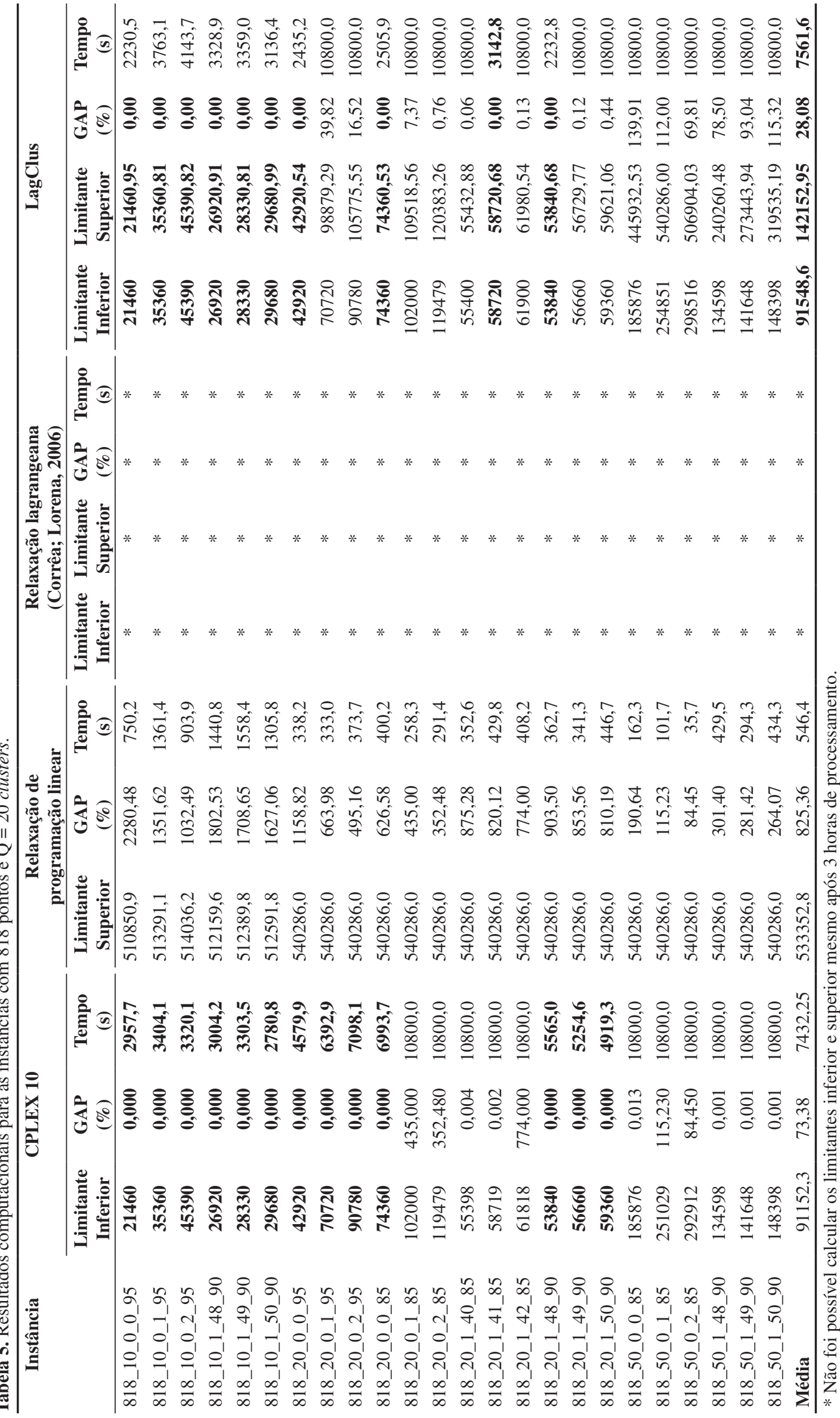




\title{
New lagrangean bounds for the probabilistic Maximal Covering Location-Allocation Problem using covering graphs
}

\begin{abstract}
The Probabilistic Maximal Covering Location-Allocation Problem (PMCLAP) aims to locate facilities maximizing the number of people served and providing a good level of service. This means that customers would not have to wait longer than the wait time established or to wait in long lines. These parameters are influenced by the number of the requests for service and service time, both probabilistic. The PMCLAP is NP-Complete and in this paper we study bounds with a Lagrangean Relaxation with Clusters (LagClus). Instead of using a conflict graph to represent a problem, in this paper another strategy for the use of LagClus using a special graph called covering graph is proposed. This approach provides interesting bounds.
\end{abstract}

Keywords: Lagrangean Relaxation. Lagrangean Relaxation with Clusters. Location Problems. Maximal Covering.

Referências Bibliográficas

ATAMTÜRK, A.; NEMHAUSER, G. L.; SAVELSBERGH, M. W. P. Conflict graphs in solving integer programming problems. European Journal of Operational Research, v. 121, n. 1, p. 40-55, 2000.

CHUNG, C. H. Recent applications of the maximal covering location planning (mclp) model. Journal of the Operational Research Society, v. 37, n. 8, p. 735-746, 1986.

CHURCH, R. L.; REVELLE, C. Maximal covering location problem. Papers of the Regional Science Association, v. 32, n. 1, p. 101-118, 1974.

CORNUÉJOLS, G.; THIZY, J. M. Some facets of the simple plant location polytope. Mathematical Programming, v. 23, n. 1, p. 50-74, 1982.

CORRÊA, F. A.; CHAVES, A. A.; LORENA, L. A. N. Hybrid heuristics for the probabilistic maximal covering location-allocation problem. Operational Research: an International Journal, v.7, n. 3, p. 323-343, 2007.

CORREAA, F. A.; LORENA, L. A. N. Aplicação da relaxação lagrangeana e do algoritmo genético construtivo na solução do problema probabilístico de localização-alocação de máxima cobertura. Revista Gestão \& Produção, v. 13, n. 2, p. 233-244, 2006a.

CORRÊA, F. A.; LORENA, L. A. N.; SENNE, E. L. F. Lagrangean relaxation with clusters for the uncapacitated facility location problem. In: CONGRESO LATINO-IBEROAMERICANO DE INVESTIGACIÓN OPERATIVA, 2006, Montevideo. Anais... Universidad de la República, 2006.

CURRENT, J. R.; O'KELLY, M. Locating emergency warning sirens. Decision Sciences, v. 23, n. 1, p. 221-234, 1981.

DASKIN, M. S. Network and discrete location: models, algorithms and applications. New York: John Wiley \& Sons, 1995.

EATON, D.; HECTOR, M.; SANCHEZ, V.; LATINGUA, R.; MORGAN, J. Determining ambulance deployment in Santo Domingo, Dominican Republic. Journal of the Operational Research Society, v. 37, n. 2, p. 113-126, 1986.

FogliatTI, M. C.; MATTOS, N. M. C. Teoria de filas. Rio de Janeiro: Interciência, 2007.

GALVÃO, R. D. Uncapacitated facility location problems: contributions. Pesquisa Operacional, v. 24, n. 1, p. 7-38, 2004.
GALVÃO, R. D.; REVELLE, C. S. A lagrangean heuristic for the maximal covering location problem. European Journal of Operational Research, v. 88, n. 1, p. 114-123, 1996.

HALE, T. S.; MOBERG, C. R. Location science research: a review. Annals of Operations Research, v. 123, n. 1-4, p. 21-35, 2003.

HOUGLAND, E. S.; STEPHENS, N. T. Air pollutant monitor sitting by analytical techniques. Journal of the Air Pollution Control Association, v. 26, p. 52-53, 1976.

ILOG. Ilog Cplex 10.0: User's manual. França, 2006. 478 p.

KARYPIS, G.; KUMAR, V. Multilevel k-way partitioning scheme for irregular graphs. Journal of Parallel and Distributed Computing, v. 48, n. 1, p. 96-129, 1998.

LARSON, R. C.; ODONI, A. R. Urban operations research. Englewood Cliffs: Prentice Hall, 1981.

LORENA, L. A. N. Análise espacial de redes com aplicações em sistemas de informações geográficas. Revista Produção, v. 3, n. 2, 2003 .

LORENA, L. A. N.; PEREIRA, M. A. A lagrangean/surrogate heuristic for the maximal covering location problem using Hillsman's edition. International Journal of Industrial Engineering, v. 9, n. 1, p. 57-67, 2002.

MARIANOV, V.; SERRA, D. Probabilistic maximal covering location-allocation models for congested systems. Journal of Regional Science, v. 38, n. 3, p. 401-424, 1998.

MARIANOV, V.; SERRA, D. Hierarchical location-allocation models for congested systems. European Journal of Operational Research, v. 135, n. 1, p. 195-208, 2001.

MOORE, G. C.; REVELLE, C. S. The hierarchical service location problem. Management Science, v. 28, n. 7, p. 775-780, 1982.

NARCISO, M. G.; LORENA, L. A. N. Lagrangean/Surrogate relaxation for generalized assignment problems. European Journal of Operational Research, v. 114, n. 1, p. 165-177, 1999.

PARKER, R. G.; RARDIN, R. L. Discrete optimization. New York: Academic Press, 1988.

PEREIRA, M. A.; LORENA, L. A. N.; SENNE, E. L. F. A column generation approach for the maximal covering location problem. International Transactions in Operations Research, v. 14, n. 4, p. 349-364, 2007. 
RIBEIRO, G. M.; LORENA, L. A. N. Lagrangean relaxation with clusters and column generation for the manufacture's pallet loading problem. Computers \& Operations Research, v. 34, n. 9 , p. $2695-2708,2007$.

RIBEIRO, G. M.; LORENA, L. A. N. Optimizing the woodpulp stowage using lagrangean relaxation with clusters. Journal of the Operational Research Society, v. 59, p. 600-606, 2008a.

RIBEIRO, G. M.; LORENA, L. A. N. Lagrangean relaxation with clusters for point-feature cartographic label placement problems.
Computers \& Operations Research, v. 35, n. 7, p. 2129-2140, 2008b.

SERRA, D.; MARIANOV, V. New trends in public facility location modeling. Barcelona, Spain: Universitat Pompeu Fabra, 2004. Economics and Business Working Paper. $\mathrm{n}^{\circ} 755$. Disponível em: $<$ http://www.econ.upf.edu/docs/papers/downloads/755.pdf $>$. Acesso em: 20 de Abril de 2008.

WOLSEY, L. A. Integer Programming. New York: John Wiley \& Sons, 1998.

\section{Sobre os autores}

\section{Francisco de Assis Corrêa}

Paulista University - UNIP

ZIP CODE 12240-420, São José dos Campos - SP, Brazil

e-mail: correa@unip.br

\section{Luiz Antonio Nogueira Lorena}

Brazilian Institute for Space Research - INPE ZIP CODE 12227-010, São José dos Campos - SP, Brazil

e-mail: lorena@lac.inpe.br

\section{Glaydston Mattos Ribeiro}

Federal University of Espírito Santo - UFES

ZIP CODE 29933-415, São Mateus - ES, Brazil

e-mail: glaydstonribeiro@ ceunes.ufes.br 